\title{
Segmenting mammographic microcalcifications using a semi-automatic procedure based on Otsu's method and morphological filters
}

\author{
Marcelo de Almeida Duarte*, André Victor Alvarenga, Carolina Maria Azevedo, Maria Julia Gregório \\ Calas, Antonio Fernando Catelli Infantosi, Wagner Coelho de Albuquerque Pereira
}

Abstract Introduction: Breast cancer has the second highest world's incidence rate, according to the Brazilian
National Cancer Institute (INCa). Clinical examination and mammography are the best methods for early
diagnosis. Computer-aided detection (CADe) and computer-aided diagnosis (CADx) systems are developed
to improve mammographic diagnosis. Basically, CADx systems have three components: (i) segmentation,
(ii) parameters extraction and selection, (iii) lesion classification. The first step for a CADx system is
segmentation. Methods: A microcalcification segmentation method is proposed, based on morphological
operators, Otsu's Method and radiologists' knowledge. Pre-processing with top-hat operators improves
contrast and reduces background noise. The Otsu's method automatically selects the best grey-level threshold
to segment microcalcifications, obtaining binary images. Following, inferior reconstruction and morphological
dilatation operators are applied to reconstruct lost structure details and fill small flaws in the segmented
microcalcifications. Finally, the Canny edge detection is applied to identify microcalcifications contour
candidates for each region-of-interest (ROI). Two experienced radiologists intervene in this semi-automatic
method, firstly, selecting the ROI and, then, analyzing the segmentation result. The method was assessed in
1000 ROIs from 158 digital images (300 dpi, 8 bits). Results: Considering the radiologists opinion, the rates
of ROIs adequately segmented to establish a diagnosis hypothesis were $97.8 \%$ for one radiologist and $97.3 \%$
for the other. Using the Area Overlap Measure (AOM) and the 2136 microcalcifications delineated by an
experienced radiologist as gold standards, the method achieved an average AOM of $0.64 \pm 0.14$, being $0.56 \pm 0.09$
for small microcalcifications and $0.66 \pm 0.13$ for the large ones. Moreover, AOM was $0.64 \pm 0.13$ for the benign
and $0.64 \pm 0.14$ for the malignant lesions with no statistical differences between them. Conclusion: Based on
these findings, the proposed method could be used to develop a CADx system that could help early breast
cancer detection.

Keywords Segmentation, Mammography, Mathematical morphology, Otsu's method, Microcalcifications, Breast cancer. 


\section{Introduction}

According to the Brazilian National Cancer Institute - INCa (Instituto..., 2012a), breast cancer has the second highest incidence rate in the world, being responsible for $22 \%$ of the new cases of cancer per year. Its incidence is growing in developed and developing countries and although most frequent in women, in 2008, it caused $125(1.05 \%)$ men deaths in Brazil. Death rates related to breast cancer in Brazil are higher, mainly due to late diagnosis, when the disease is already in advanced stage (Instituto..., 2012a). Also according to INCa (Instituto..., 2012b), early detection and tumour removing in an initial phase are the more efficient strategies to reduce death rates.

Clinical and mammographic examinations have been considered the best methods to find early signs of breast cancer. The mammographic exam aims at identifying non-palpable breast lesions (Instituto..., 2012b). Nevertheless, several factors, such as the physicians' knowledge and experience, the equipment quality and the presence of adipose and glandular tissues types, may interfere in the accuracy of mammograms, making diagnosis difficult. Even if mammography is carried out with adequate equipment and by an experienced technician, the final exam quality is highly dependent on the breast tissue itself, as the more adipose it is, the easier to analyse and diagnose (Azevedo, 1994). Barlow (2002) reported a large number of errors and divergent results in mammographic exams made in USA, mainly due to the radiologists' inexperience, besides the already mentioned difficulties.

Microcalcifications have been considered a relevant sign of malignancy since they are present in a great number of malignant lesions. Based on histological examination, $60 \%$ to $80 \%$ of the carcinomas reveal the presence of microcalcifications although they are detected in just $30 \%$ to $50 \%$ of mammograms (Halkiots et al., 2007). The difficulty of detecting microcalcifications in mammograms is due to their variation in shape (from granular to rod shapes), orientation, brightness, diameter size and also the surrounding tissue (Wei et al., 2009). In an effort to help in microcalcifications diagnosis, Computer-Aided Detection (CADe) and Computer-Aided Diagnosis (CADx) systems have been developed (Elter and Horsch, 2009; Nishikawa, 2007). Such systems are projected to help minimising the false-positive and false-negative rates of breast cancer diagnosis, and are usually based on parameters extracted from microcalcifications (De Santo et al., 2003; Veldkamp et al., 2000).
CADe systems aim at automatic detecting lesions (including microcalcifications), while CADx are used to perform their classification. These latter systems are generally used as a second opinion, aiming at increasing the accuracy of radiologists' final decision (Calas et al., 2012; Chen and Lee, 1997; Cheng et al., 2003; Elter and Horsch, 2009; Jalalian et al., 2013; Nishikawa, 2007). CADx are basically composed of three steps: (i) segmentation, (ii) parameters extraction and selection, (iii) lesions classification. Although the individual improvement of any of them could enhance the whole system performance, the segmentation is considered a very important step in CADx systems, since it defines the microcalcifications characteristics to be used in the next CADx steps (Arikidis et al., 2010; Paquerault et al., 2004; Timp and Karssemeijer, 2004).

Several techniques have been used to segment microcalcifications, such as active contours (Arikidis et al., 2008, 2010; Paquerault et al., 2004), grey-level histogram features associated to fuzzy rule-based classifiers (Gravielides et al., 2002), methods based on image entropy (Mohanalin et al., 2009), wavelet analysis (Chen and Lee, 1997) and morphological filters (Halkiots et al., 2007; Stojic et al., 2006). Despite the various methods presented in literature, segmenting individual microcalcifications is still an issue to be addressed (Arikidis et al., 2010).

This work presents a microcalcification segmentation semi-automatic method, based on morphological operators and Otsu's Method (Otsu, 1979). The method is applied to 1000 Regions of interest (ROIs) from images provided by the Digital Database for Screening Mammography (DDSM) - University of South Florida (University..., 2012). As a pre-processing procedure, top-hat morphological operators are used to enhance the image contrast between microcalcifications and background, while Otsu's Method automatically selects the best grey-level threshold to segment microcalcifications. As a post-processing stage, a set of morphological filters is applied to fill up any small flaw in the segmented microcalcifications. The proposed method incorporates expert's knowledge by considering the opinion of the radiologist about the segmented images. The radiologist evaluates if it is possible to formulate an adequate diagnosis hypothesis with the segmented images. In this work, for evaluating the method, the opinion of two experienced radiologists was independent and jointly considered. A quantitative evaluation approach, based on Area Overlap Measure, was also carried out to evaluate the results. 


\section{Methods}

In this section, we firstly describe the used database, then the method developed to segment the microcalcifications present in a ROI and finally, the evaluation methodologies of the results.

\section{The database}

The database is composed of 158 mammograms from 78 patients, extracted from three image sets of the DDSM database: benign_1, benign_2 and cancer_1. Similarly to other authors (De Santo et al., 2003; Fu et al., 2005; Stojic et al., 2005), in order to reduce the computational time, the number of bits of these images ( $300 \mathrm{dpi})$, originally 12 or 16 , were reduced to 8 bits. Moreover, 1000 regions of interest (ROIs) up to $41 \times 41$ pixels (Arikidis et al., 2008) were selected by the double reading procedure. First, a ROI was chosen by an experienced radiologist and then independently confirmed by another. Five hundred ROIs were selected from images of the benign databases (793 microcalcifications) and another 500 from the cancer database (1343 microcalcifications). All ROIs were from delimited regions, already marked in the DDSM images (University..., 2012). The microcalcifications were also classified by their maximum diameters, following the criterion proposed by Arikidis et al. (2010). Thus, the small lesions (608 microcalcifications) were those with maximum diameters smaller than $460 \mu \mathrm{m}$, otherwise they were classified as large lesions (1528 microcalcifications). An example of a ROI extracted from a mammogram
(Figure 1a) with malignant large microcalcifications is depicted in Figure $1 b$.

\section{Segmentation procedure}

In order to improve the contrast and reduce the background noise of the DDSM images, a pre-processing procedure has been used. Firstly, to enhance structures smaller than the structuring element (SE), a top-hat by opening by reconstruction was applied to the original ROI. Due to differences in the sizes and shapes of microcalcifications, this morphological operator was always applied to the original ROI, using three different SEs, that is, a cross-shaped SE of 3-pixel size, a disc-shaped SE of 5-pixel diameter, and a disc-shaped SE of 17-pixel diameter, resulting in three different images, here called "thor". Then, to remove the structures that present the highest grey-levels and also to enhance the background noise, these resulting images were filtered by a top-hat by closing by reconstruction with a disc SE of 51-pixel diameter, resulting in images here called "thcr". Finally, a point-wise subtraction between the images "thor" and "thcr" was performed and the "diff" images were obtained. In these images, the possible microcalcifications are emphasised whilst the background noise is almost totally removed. All SEs used in this procedure have been heuristically chosen.

The pre-processing technique is illustrated in Figure 1. In this case, the original ROI (Figure 1b) was extracted from a medium lateral oblique view of a digital mammogram (Figure 1a). Firstly, the top-hat
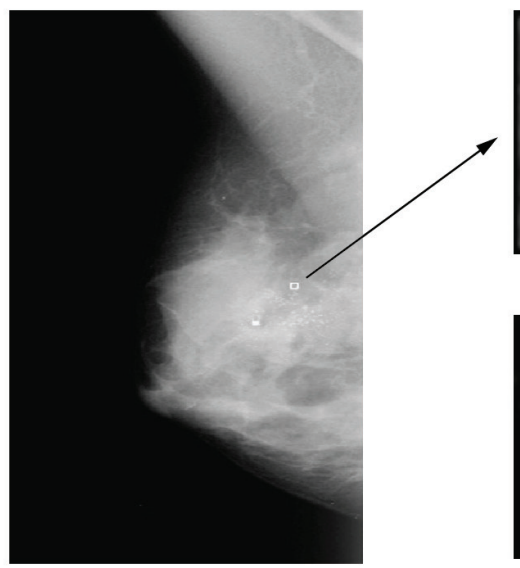

(a)

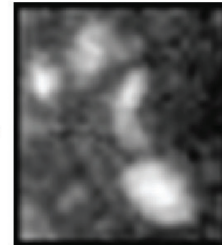

(b)

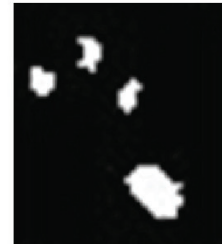

(f)

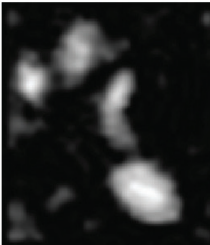

(c)

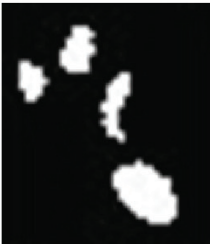

(g)

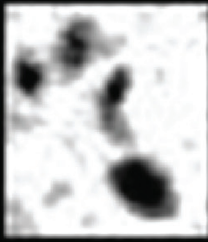

(d)

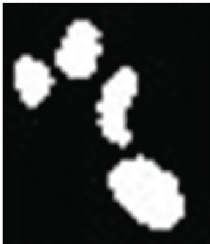

(h)

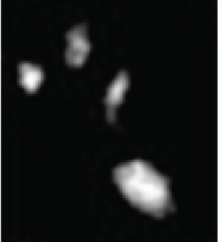

(e)

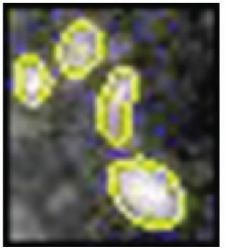

(i)

Figure 1. (a) Medium lateral oblique view of a digital mammogram and a selected ROI (white square selected by a radiologist in the mammogram). (b) Original ROI extracted from (a) (white square), augmented. (c) Image "thor" obtained from the top-hat by opening by reconstruction application in (b). (d) Image "thcr" resulted from the top-hat by closing by reconstruction applied to (c). (e) "diff" image obtained from the point-wise subtraction between "thor" and "thcr". (f) Binary image from "diff”, after applying Otsu's Method. (g) Inferior reconstruction applied in (f). (h) Morphological dilatation applied in (g). (i) Segmented image. 
by opening by reconstruction with a disc-shaped SE of 17-pixel diameter was applied, resulting the image depicted in Figure 1c. This latter image was then filtered by the top-hat by closing by reconstruction (Figure 1d). After performing the point-wise subtraction between "thor" and "thcr" images, the possible microcalcifications (white structures) are emphasised whilst the background noise is almost totally removed (Figure 1e).

Following the pre-processing procedure, the Otsu's method was applied to the "diff" images. Hence, by automatically determining the grey-level threshold, binary images were obtained, as the one illustrated by Figure 1f. It is worth emphasizing that the Otsu's method assumes that any image contains two classes of pixels (e.g. foreground and background) and calculates the optimum threshold separating those two classes so that their combined spread (intra-class variance) is minimal (Otsu, 1979).

As a post-processing procedure, an inferior reconstruction operator was applied to the binary images (disc SE with 3-pixel diameter) to reconstruct possible lost structure details (illustrated in Figure 1g). The binary images were used as mark, while the "diff" images were set as the reference mask for the reconstruction. Then, a morphological dilatation (cross SE with 3-pixel size) was applied to fill any small flaw in the segmented microcalcifications (exemplified by Figure 1h). Finally, the canny edge detection was applied to identify microcalcifications contours candidates for each ROI (illustrated in Figure 1i). Similarly to the pre-processing, here the SEs were also heuristically determined.

The segmentation method was implemented in MATLAB $^{\circledR}$ (Mathworks Inc., Natick, MA) using the SDC Morphology Toolbox V1.6 (SDC Information Systems, Naperville, USA).

\section{Evaluating the segmentation methodology}

In a qualitative evaluation, two experienced radiologists (more than 30 and 15 years of practice, respectively) independently analyse the results. According to Cheng et al. (2003), radiologists' opinions are well accepted for evaluating segmentations methods. This evaluation procedure has been carried out nearly two months after the ROIs double reading selection procedure previously described. For each of the 1000 selected ROIs, it was created a figure depicting the original ROI and their three correspondent segmented images (one for each SE for the top-hat by opening by reconstruction operator used in the pre-processing procedure), containing the microcalcifications contours candidates. These figures were then presented independently and randomly to both radiologists.
For each figure, the radiologist should choose, among the three segmented images, the one considered as the best for elaborating an adequate diagnosis hypothesis. If he is not able to elaborate a diagnosis hypothesis with any of them, none of the segmented images is considered adequate.

A quantitative evaluation process was carried out independently of the qualitative one. The most experienced radiologist manually delineated (that is, segmented) the 2136 microcalcifications of the 1000 selected ROIs and these contours were taken as gold standards for calculating the Area Overlap Measure (AOM), expressed by (Arikidis et al., 2010):

$\mathrm{AOM}=\frac{\operatorname{area}(M \cap R)}{\operatorname{area}(M \cup R)}$

where $\mathrm{M}$ is the microcalcification manually delineated by the radiologist and $\mathrm{R}$ represents the segmentation obtained with the proposed method (that is, considered by the radiologist as the best one for elaborating an adequate diagnosis hypothesis). The symbol $\cap$ is the intersection, that is, the number of pixels common to both segmentation procedures and the symbol $\cup$ represents the union of the $\mathrm{M}$ and $\mathrm{R}$ areas. Hence, if there is no overlap between the delineated microcalcification and the one from the proposed method, $\mathrm{AOM}=0$. For a complete overlap, $\mathrm{AOM}=1$.

The Wilcoxon non-parametric statistical test for paired data (Wilcoxon two-sided rank sum test, $\mathrm{p}$-values $<0.05$ ) was performed to evaluate if there were significant differences between the results of AOMs from benign and malignant microcalcifications, and from small and large ones.

\section{Results}

Figure 2 depicts three original ROIs and respective segmented images obtained by using the different SEs described in the pre-processing procedure (top-hat by opening by reconstruction operator). For the sake of comparison, the microcalcifications manually delineated by the most experienced radiologist for each original ROI are also shown. According to both radiologists' opinion, the most adequate segmentation was achieved by applying a 3-pixel diameter cross-shaped SE (Figure 2c) to the ROI of Figure 2a. On the other hand, for a different ROI (Figure 2f), they considered the image segmented with the disc-shaped SE with 5-pixel diameter (Figure 2i) as the most adequate one. Furthermore, for the ROI depicted in Figure 2k, the most adequate segmentation (Figure 2o) was achieved by applying the disc-shaped SE with 17-pixel diameter. 
For the 1000 selected ROIs, one of the radiologists indicated at least one adequate segmented image, among the three presented, in $97.8 \%$ of them, independently of being extracted from the benign or malignant image databases (Table 1). For the other radiologist, this value was $97.3 \%$. Taking into account only the 500 ROIs extracted from the benign databases, the rates were $98.0 \%$ for one of the radiologists and $97.8 \%$ for the other. For 500 ROIs selected from the cancer database, these rates were $97.6 \%$ and $96.8 \%$, respectively.

Taking the radiologists' opinion separately (Table 2), the 17-pixel diameter disc-shaped SE resulted in adequate microcalcifications segmentation for at least $74.3 \%$ of the ROIs. The resulting segmentation with the 5-pixel diameter disc-shaped SE was considered adequate for at least $20.2 \%$ of the ROIs, while for the 3-pixel size cross-shaped SE this figure was at least $0.3 \%$. In the worst case, the second radiologist has considered that microcalcifications presented in $2.7 \%$ of the ROIs were inadequately segmented, independent of the SE used.

Considering the joint radiologists' opinion (Table 3), there is an agreement between them that the microcalcification segmentation method proposed here was adequate for 966 ROIs ( $96.6 \%$ ), that is, both radiologists considered themselves able to formulate a diagnosis hypothesis with at least one of the three resulting segmented images. Also for ROIs with bright (Figure 3a) and dark (Figure 3b) backgrounds and close to artifacts (Figure 3c), according to the radiologists' opinion, the method was capable to adequately

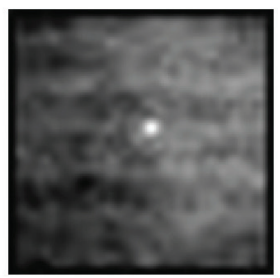

(a)

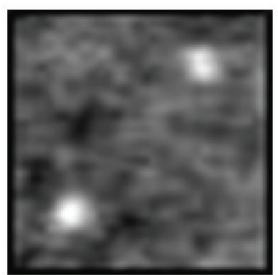

(f)

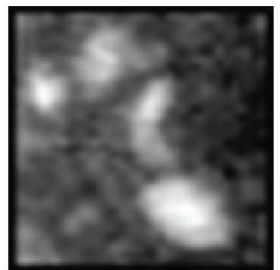

(k)

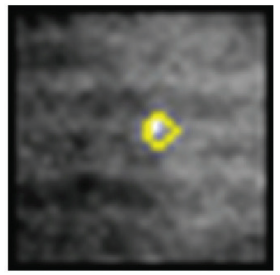

(b)

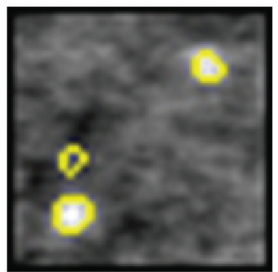

(g)

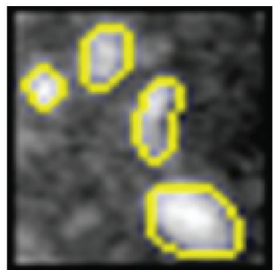

(1)

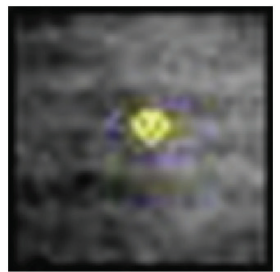

(c)

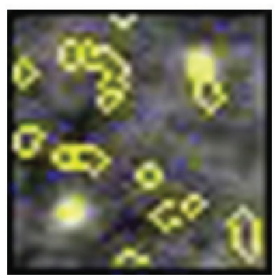

(h)

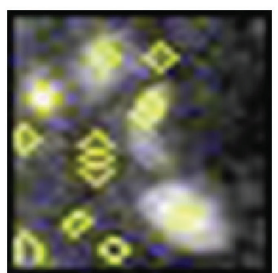

(m)

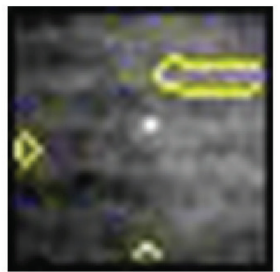

(d)

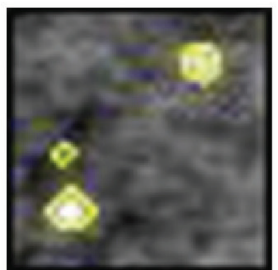

(i)

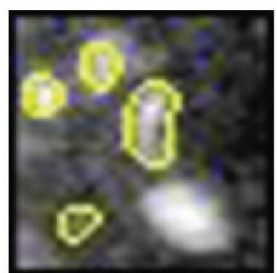

(n)

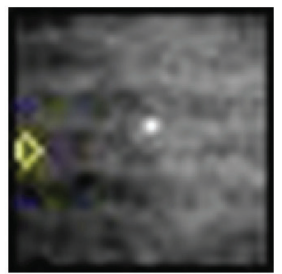

(e)

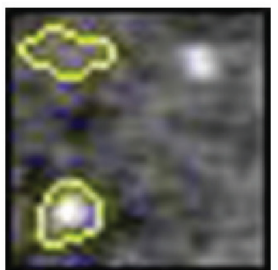

(j)

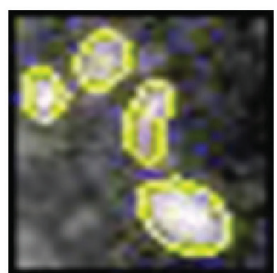

(o)

Figure 2. (a), (f) and (k) Original ROIs. (b), (g) and (l) their respective microcalcifications manually delineated by the most experienced radiologist. Respective segmentation results obtained by using the cross-shaped SE with a 3-pixel size (c), (h) and (m); disc-shaped SE with a 5-pixel diameter (d), (i) and (n) and disc-shaped SE with a 17-pixel diameter (e), (j) and (o). For ROI (a), both radiologists indicated (c) as the most adequate to formulate a diagnosis hypothesis. They also indicated (i) and (o) as the most adequate for (f) and (k), respectively.

Table 1. Rates of adequate (AD) and inadequate (INAD) microcalcifications segmentation images, according to radiologists' opinion, in ROIs extracted from images of the benign and malignant databases.

\begin{tabular}{cccccccc}
\hline & $\begin{array}{c}\text { AD } \\
\text { (benign) }\end{array}$ & $\begin{array}{c}\text { INAD } \\
\text { (benign) }\end{array}$ & $\begin{array}{c}\text { AD } \\
\text { (malignant) }\end{array}$ & $\begin{array}{c}\text { INAD } \\
\text { (malignant) }\end{array}$ & $\begin{array}{c}\text { AD } \\
\text { (total) }\end{array}$ & $\begin{array}{c}\text { INAD } \\
\text { (total) }\end{array}$ & Total \\
\hline \multirow{2}{*}{$\mathbf{1}^{\text {st }}$ radiologist } & 490 & 10 & 488 & 12 & 978 & 22 & 1000 \\
\hline \multirow{2}{*}{$\mathbf{2}^{\text {nd }}$ radiologist } & $(98.0 \%)$ & $(2.0 \%)$ & $(97.6 \%)$ & $(2.4 \%)$ & $(97.8 \%)$ & $(2.2 \%)$ & \\
& $(97.8 \%)$ & $(2.2 \%)$ & $(96.8 \%)$ & $(3.2 \%)$ & $(97.3 \%)$ & $(2.7 \%)$ & 1000 \\
\hline
\end{tabular}


Table 2. Results of radiologists' opinion separated by SE used to achieve an adequate segmentation for a diagnosis hypothesis.

\begin{tabular}{cccccc}
\hline & $\begin{array}{c}\text { 3-pixel } \\
\text { cross-shaped }\end{array}$ & $\begin{array}{c}\text { 5-pixel } \\
\text { disc-shaped }\end{array}$ & $\begin{array}{c}\text { 17-pixel } \\
\text { disc-shaped }\end{array}$ & Not Adequate & Total \\
\hline $\begin{array}{c}\mathbf{1}^{\text {st }} \\
\text { radiologist }\end{array}$ & 3 & 202 & 773 & 22 & 1000 \\
\hline $\begin{array}{c}\mathbf{2}^{\text {nd }} \\
\text { radiologist }\end{array}$ & $(0.3 \%)$ & $(20.2 \%)$ & $(77.3 \%)$ & $(2.2 \%)$ & 1000 \\
\hline
\end{tabular}

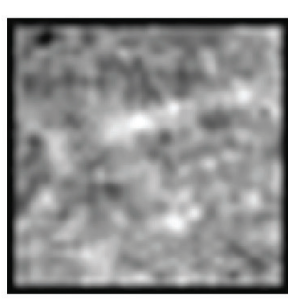

(a)

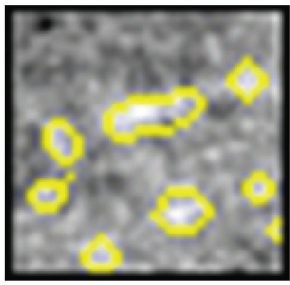

(d)

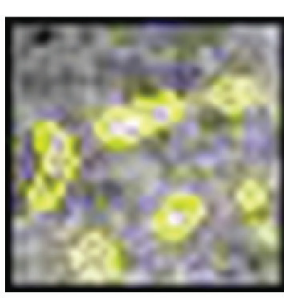

(g)

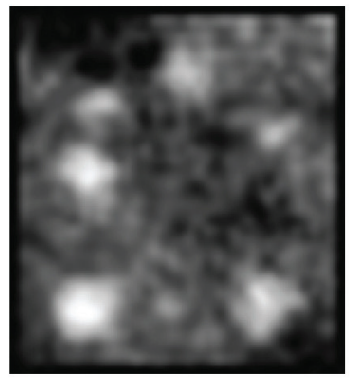

(b)

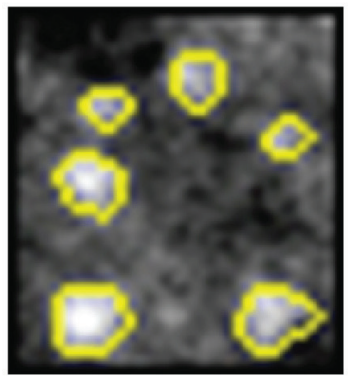

(e)

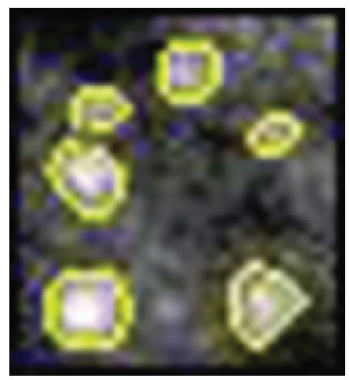

(h)

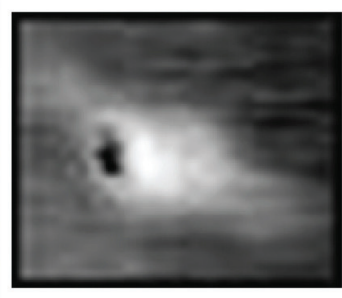

(c)

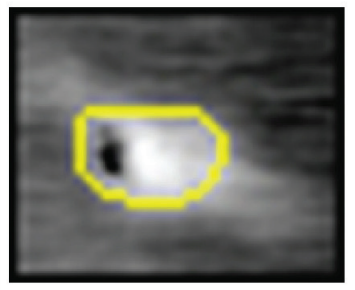

(f)

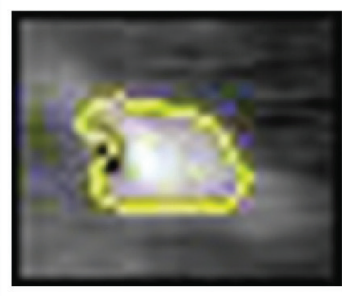

(i)

Figure 3. Examples of original ROIs presenting microcalcifications over (a) bright and (b) dark backgrounds, and (c) nearby an artifact. (d), (e) and (f): respective microcalcifications manually delineated by the most experienced radiologist. Segmented images considered adequate by both radiologists: (g) with the 5-pixel diameter disc-shaped SE, (h) and (i), both with the 17-pixel diameter disc-shaped SE.

segment the microcalcifications (Figures $3 \mathrm{~g}, 3 \mathrm{~h}$ and $3 \mathrm{i}$, respectively), although with different SE. On the other hand, for fifteen ROIs (1.5\%), both radiologists considered the resulting segmentations, independent of the SE used, as inadequate (e.g. Figures 4a and 4f). For another nineteen ROIs (1.9\%), the radiologists disagreed in indicating the same segmented image (e.g. Figures 5a and 5f).

Based on the 2136 microcalcifications (from the 1000 selected ROIs) manually delineated by the most experienced radiologist, the average Area Overlap Measure (AOM) was estimated as $0.64 \pm 0.14$. For the 793 benign lesions, $\mathrm{AOM}=0.64 \pm 0.13$ while for the 1343 malignant ones, $\mathrm{AOM}=0.64 \pm 0.14$. Furthermore, there is no significant difference between the AOM of malignant and benign microcalcifications $(\mathrm{p}=0.81$, Wilcoxon non-parametric statistical test for paired data). On the other hand, there is statistical significant difference between small (AOM $=0.56 \pm 0.09$ for 608 lesions with maximum diameters smaller than 
$460 \mu \mathrm{m})$ and large $(\mathrm{AOM}=0.66 \pm 0.13$ for 1528 lesions) microcalcifications ( $\mathrm{p}<0.0001)$.

The cumulative distribution curves of the AOM for each case can be observed in Figures 6 and 7. In
Figure 6 one can see that for all microcalcifications (full line), more than $50 \%$ have AOM higher than 0.7 , while at least $80 \%$ have AOM higher than 0.6. In this same figure, it can be seen that the curves for

Table 3. Rates of agreement between both radiologists, regarding segmentation adequacy for a possible diagnosis hypothesis.

\begin{tabular}{cccc}
\hline Results & $\begin{array}{c}\text { Adequate } \\
\left(\mathbf{1}^{\text {st }} \text { radiologist }\right)\end{array}$ & $\begin{array}{c}\text { Not Adequate } \\
\left(\mathbf{1}^{\text {st }} \text { radiologist }\right)\end{array}$ & Total \\
\hline $\begin{array}{c}\text { Adequate } \\
\left(2^{\text {nd }} \text { radiologist }\right)\end{array}$ & $966(96.6 \%)$ & $7(0.7 \%)$ & $973(97.3 \%)$ \\
\hline $\begin{array}{c}\text { Not Adequate } \\
\left(2^{\text {nd }} \text { radiologist }\right)\end{array}$ & $12(1.2 \%)$ & $15(1.5 \%)$ & $27(2.7 \%)$ \\
\hline Total & $978(97.8 \%)$ & $22(2.2 \%)$ & $1000(100 \%)$ \\
\hline
\end{tabular}

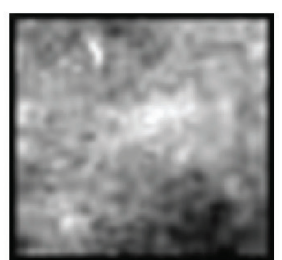

(a)

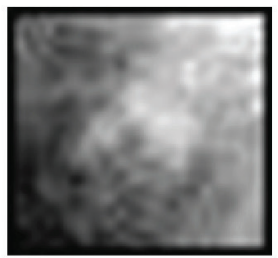

(f)

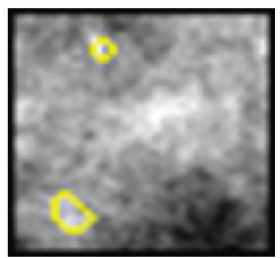

(b)

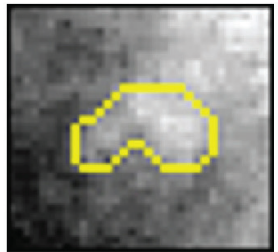

(g)

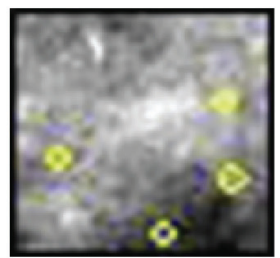

(c)

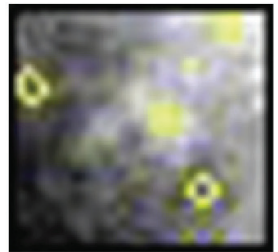

(h)

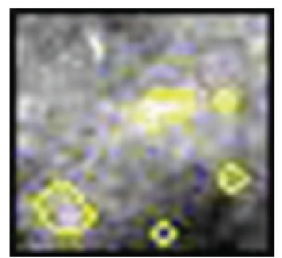

(d)

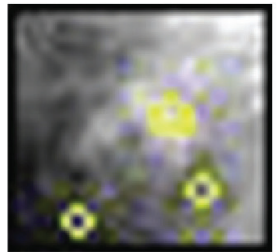

(i)

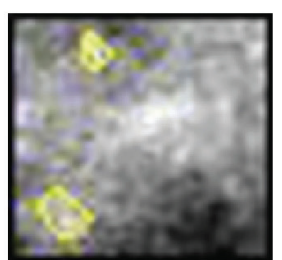

(e)

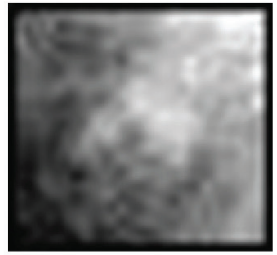

(j)

Figure 4. Examples of original ROIs (a) and (f) for which both radiologists considered segmentation to be inadequate. (b) and (g) respective microcalcifications manually delineated by the most experienced radiologist; (c) and (h) respective segmented images using the cross-shaped SE with a 3-pixel size; (d) and (i) disc-shaped SE with a 5-pixel diameter; and (e) and (j) disc-shaped SE with a 17-pixel diameter.

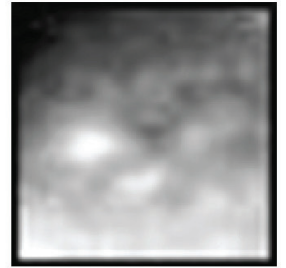

(a)

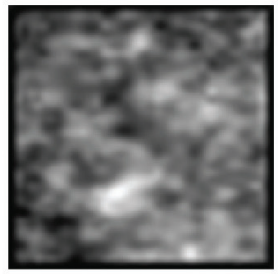

(f)

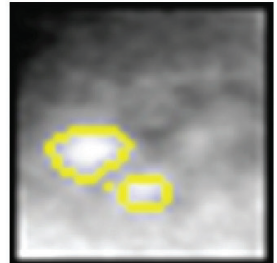

(b)

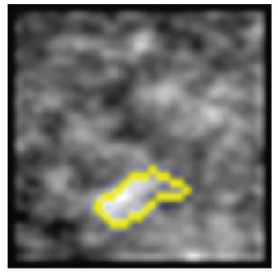

(g)

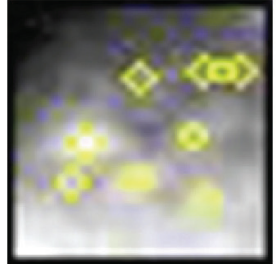

(c)

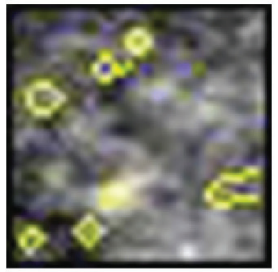

(h)

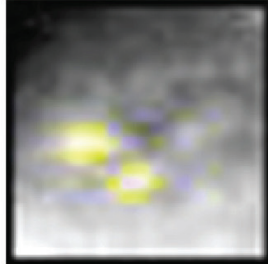

(d)

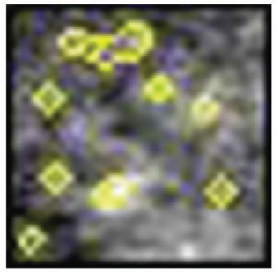

(i)

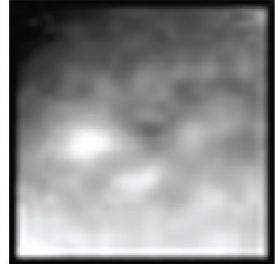

(e)

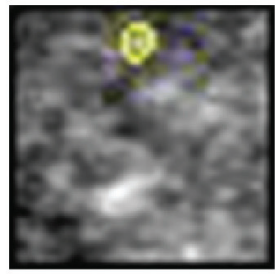

(j)

Figure 5. Examples of ROIs (a) and (f) for which the radiologists disagreed about the adequacy of the segmented images using: (c) and (h) the cross-shaped SE with a 3-pixel size, (d) and (i) disc-shaped SE with a 5-pixel diameter and (e) and (j) disc-shaped SE with a 17-pixel diameter. Images (b) and (g) are the microcalcifications manually delineated by the most experienced radiologist. For (a), this radiologist chose (d) as adequate while the other chose none of them. For (b), no segmentation was chosen by this radiologist, while the other pointed out (i) as adequate for a diagnosis hypothesis. 


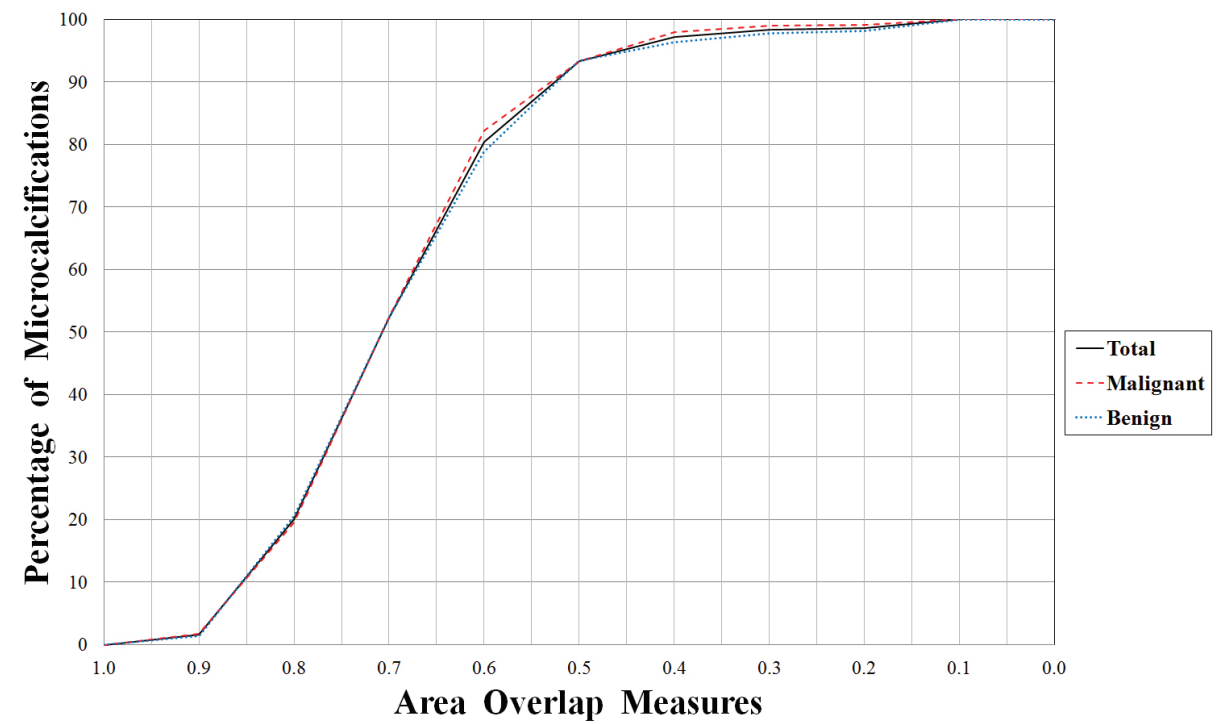

Figure 6. Cumulative distribution curves of the Area Overlap Measures for the MM method, considering (full line) all microcalcifications; (dashed) malignant microcalcifications; (dotted) benign microcalcifications.

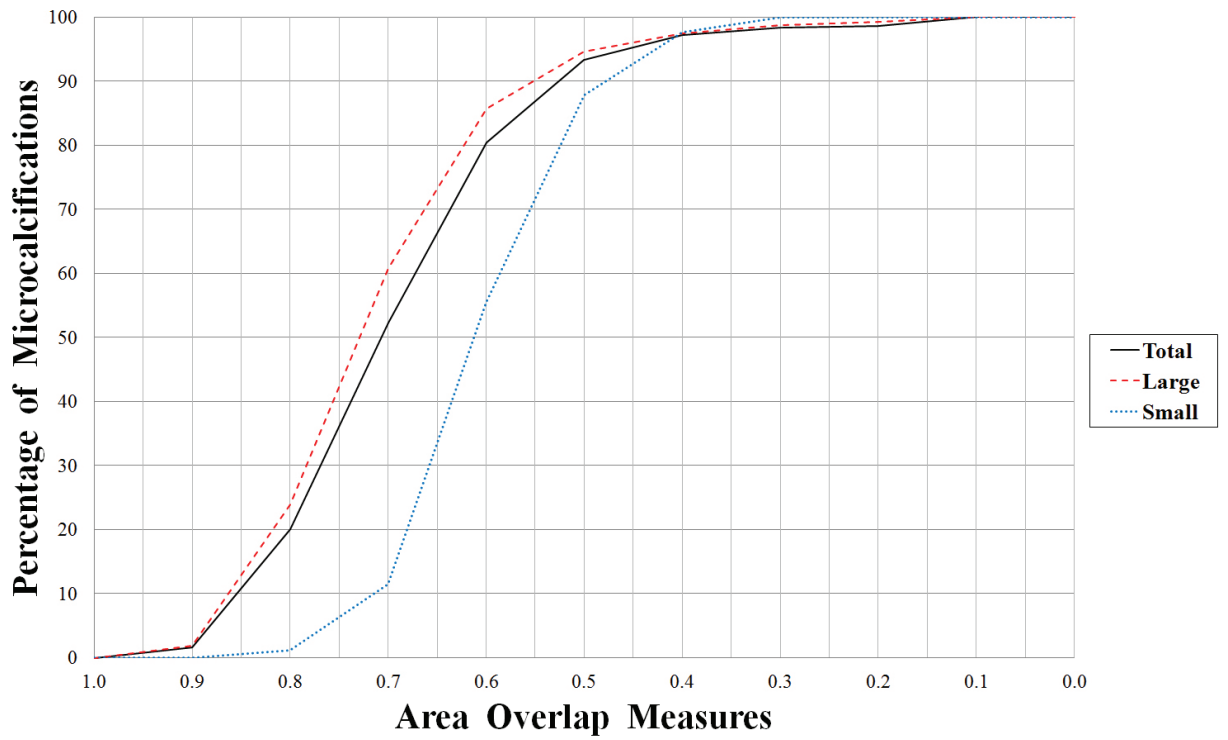

Figure 7. Cumulative distribution curves of the Area Overlap Measures for the MM method, considering (full line) all microcalcifications; (dashed) large microcalcifications; (dotted) small microcalcifications.

malignant and benign microcalcifications are similar. In Figure 7, note that cumulative distribution curve of $\mathrm{AOM}$ is higher for large microcalcifications.

\section{Discussion}

This work was focused on the first stage of a CADx system, which is segmenting microcalcifications independently of their sizes and shapes. With this aim, the proposed method, based on Otsu's Method and morphological operators filtering, always uses three different SEs for the top-hat by opening by reconstruction operator applied to the ROIs in the beginning of the pre-processing procedure. For each ROI, three segmented images (one for each used SE size and shape) are presented to the radiologist who chooses the image segmentation that allows elaborating the diagnosis hypothesis.

According to the radiologists' opinion (Table 2), $77.3 \%$ (radiologist \#1) and 74.3\% (\#2) of the ROIs 
had microcalcifications adequately segmented by using the 17-pixel disc-shaped SE. On the other hand, the adequacy rates were $20.2 \%$ (\#1) and 22.1 (\#2) by employing the 5-pixel diameter disc-shaped SE and $0.3 \%$ (\#1) and $0.9 \%$ (\#2) for the 3-pixel cross-shaped SE. These results indicate the importance of using SE of different sizes and shapes to segment different microcalcifications. Moreover, the importance of making the right choice of the SE can also be observed qualitatively in Figure 3, where microcalcifications of different sizes and also a ROI containing an artifact (Figure 3c) are properly segmented (Figure 3i).

Herein, the proposed segmentation procedure could not, in some cases, segment all individual microcalcifications in a ROI. Nevertheless, considering the radiologists' knowledge, those cases were still considered adequate since radiologists agreed about their diagnosis based on the segmented images. Taking into account the radiologists' opinion independently, the resulting segmentations were considered inadequate to perform a diagnosis for no more than $2.2 \%$ of the benign lesions and 3.2\% for the malignant ones. This rate was $2.7 \%$, considering all microcalcifications (Table 1). Moreover, considering the joint radiologists' opinion about the adequacy (Table 3 ), they felt comfortable to indicate at least one segmented result as adequate to formulate a diagnosis hypothesis for $96.6 \%$ of the ROIs. They pointed out just fifteen ROIs $(1.5 \%)$ as not adequately segmented for this purpose (e.g. Figure 4). For nineteen ROIs (1.9\%), the radiologists disagreed in indicating the same segmented image (e.g. Figure 5). In all these 34 ROIs, it was noticeable significant background noise (e.g. Figures 4a, 4f, 5a and 5f), and very small microcalcifications (e.g. Figures $4 \mathrm{a}$ and $5 \mathrm{f}$ ).

Duarte et al. (2010a, 2010b) used a different semi-automatic procedure to perform the segmentation of microcalcifications and their clusters present in ROIs from images of a different database (13 digital mammograms from eight INCa patients and 41 digitalized ones from 12 patients of the Gaffrée \& Guinle University Hospital). In these works, images were segmented according to the grey-levels thresholds extracted from their morphological filtered grey-levels histogram (the thresholds were taken as the peaks of the filtered histogram). A set containing several segmented images, one for each threshold, was presented to the radiologists and, in some cases, they had to examine more than 10 segmented ROIs candidates to choose just one as the best result, spending a long time to complete the entire task. In the present work, the segmentation method was improved by using the Otsu's Method to automatically determine the grey-level threshold for the three images generated by the application of the three SEs in the top-hat by opening by reconstruction operator, in the pre-processing procedure. Hence, the radiologists can simply decide whether the segmentation is adequate or not for diagnosis purposes, just observing three different segmented images, demonstrating a clear evolution of the method.

Moreover, the rates of ROIs with microcalcifications considered as adequately segmented to establish a diagnosis hypothesis, according to two experienced radiologists, were $97.8 \%$ for one radiologist and $97.3 \%$ for the other (Table 1). These rates were higher than those $(91.9 \%$ and $88.6 \%$ to each radiologist) achieved by Duarte et al. (2011), in which the same method proposed here was assessed with a different database (54 mammograms from INCa and Gaffrée \& Guinle University Hospital patients). In this case, 236 ROIs were processed (also $300 \mathrm{dpi}, 8 \mathrm{bits}$ ), containing malignant (104 ROIs) and benign (132 ROIs) microcalcifications.

Paquerault et al. (2004) also compared, qualitatively, the results obtained in the application of two microcalcifications segmentation methods: a Radial Active Contours method (RAC), based on gradients, and a Region-Growing method (RG). The authors used a database with mammograms of 76 patients, selected from the archives of the Department of Radiology at the University of Chicago - US, containing 144 microcalcifications clusters. The evaluation was performed by two observers ( $\mathrm{O} 1$ and $\mathrm{O} 2)$ who were instructed to rate the accuracy of the segmentation based on how well the segmented contour agreed with their mentally visualized contours. The accuracy rates were $67.9 \pm 22.9(\mathrm{O} 1)$ and $31.4 \pm 24.5(\mathrm{O} 2)$ for the RG method, and $91.7 \pm 3.2(\mathrm{O} 1)$ and $83.2 \pm 12.4(\mathrm{O} 2)$, for the RAC. Hence, we have chosen the minimum rate for the RAC method by Paquerault et al. (2004) $(83.2-12.4=70.8)$ to classify a segmented image as adequate, which results in considering $97.3 \%$ of the ROIs as adequately segmented. This finding suggests an efficacy at least similar to the RAC method proposed by Paquerault et al. (2004). The authors also implemented a third technique, based on the Watershed transform, but have not compared the accuracy rates obtained with this technique. It is important to quote that in these authors' work, all segmentation methods begin with manually identified seed points, which were carefully selected to represent the centers of the individual microcalcifications. In the method here proposed, the seed point is never required.

Using only cranial-caudal images from the DDSM database, Moradmand et al. (2011) applied morphological operators and wavelet transformation to enhance the mammography images contrast, 
before investigating two semi-automatic methods of microcalcifications segmentation: Adaptive Threshold and Watershed Segmentation. The authors conclude, based on a qualitative radiologists' evaluation, that Watershed segmentation method is more accurate than the Adaptive Threshold one, although being more time consuming.

By observing the cumulative distribution curves of the Area Overlap Measures in Figure 6, it can be seen that more than $50 \%$ of the 2136 microcalcifications (actually, 52.3\% = 1117 microcalcifications) have AOM higher or equal than 0.7 and at least $80 \%$ $(1719$ microcalcifications $=80.5 \%)$ have $\mathrm{AOM}$ higher or equal than 0.6 (full line curve). A similar behavior can be seen for the benign (dotted) and malignant (dashed) microcalcifications. Figure 7 shows that approximately $11 \%$ of the small (dotted) microcalcifications $(11.5 \%=70$ microcalcifications $)$ have AOM higher or equal than 0.7 and at least $55 \%$ $(377$ microcalcifications $=55.4 \%)$ have $\mathrm{AOM}$ higher or equal than 0.6 . For the large microcalcifications (dashed), AOM was higher or equal than 0.7, approximately, for $60 \%$ of them $(60.8 \%=929$ microcalcifications) and it was higher or equal than 0.6 for at least $85 \%$ (1311 microcalcifications $=85.8 \%)$.

Considering these results, we observed that the proposed method performed equally, independent of the kind of lesion (benign or malignant). Additionally, considering that microcalcifications shapes use to be determinant for the classification of lesion types (Jalalian et al., 2013; Paquerault et al., 2004; Veldkamp et al., 2000), these results suggest that microcalcifications shapes were not impediments for this method to segment any kind of microcalcification. However, the method is sensible to the microcalcifications sizes.

Using the same database, but not exactly the same images, Arikidis et al. (2010) presented a method based on Active Contours to segment microcalcifications, which also employs radiologists' intervention. In that work, the seed to initialise the Active Contours should be manually defined by a radiologist, and a ROI is defined as a square of $81 \times 81$ pixels, centered at the seed point. Hence, based on this scheme, their method will segment only the microcalcifications individually marked by the radiologist, which tends to be time consuming. The authors reported that the computational time for segmenting each microcalcification was $0.42 \mathrm{~s}$. Arikidis et al. (2010) assessed the results by comparing the obtained segmentation areas with the ones manually defined by a radiologist, and pointed out an AOM mean value of $0.61 \pm 0.16$ for large microcalcifications and $0.61 \pm 0.14$ for the small ones. In our proposed method, this result was a slightly higher for the large microcalcifications $(0.66 \pm 0.13)$, but a little smaller $(0.56 \pm 0.09)$ for the small lesions. Arikidis et al. (2010) did not present results based on the microcalcifications types.

In the proposed method, the computation time was $0.23 \mathrm{~s}$ to segment each ROI, independent of the number of microcalcifications, which could be up to 13. For this extreme case, the method described by Arikidis et al. (2010) would take $5.46 \mathrm{~s}$ to process all the 13 microcalcifications and hence some 23 times the computation time of the method here proposed.

Different from Paquerault et al. (2004) and Arikidis et al. (2010) methods, where the radiologists' intervention is required in an intermediate stage of the segmentation procedure (e.g., introducing a seed point to represent the microcalcification center), the proposed method integrates the radiologists' knowledge in a key step of the process, choosing the image segmentation that allows elaborating the diagnosis hypothesis. As a result of this synergy, the procedure applied to the studied ROIs was able to adequately segment more than $97 \%$ of the microcalcifications.

Overall, this paper presents a semi-automatic method to segment microcalcifications from mammographic images, which incorporates the radiologists' opinion in the final stage of the segmentation method. In the tested database, according to two experienced radiologists, the rates of ROIs with microcalcifications considered as adequately segmented were $97.8 \%$ for one radiologist and $97.3 \%$ for the other. In a quantitative evaluation, the average Area Overlap Measure was $0.64 \pm 0.14$, considering all 2136 microcalcifications delineated. There were no statistical differences between the AOMs calculated for the benign and the malignant lesions. These results encourage us to use the proposed segmentation method in extracting parameters from the segmented microcalcifications and clusters, intending to develop a classifier algorithm to discriminate malignant and benign ones, which could integrate a CADx system.

\section{Acknowledgments}

The authors acknowledge the Brazilian agencies CNPq, CAPES and FAPERJ for the financial support, and the Gama Filho University for technical and partial financial support.

\section{References}

Arikidis NS, Skiadopoulos S, Karahaliou A. B-spline active rays segmentation of microcalcifications in mammography. Medical Physics. 2008; 35(11):5161-71. http://dx.doi. org/10.1118/1.2991286 
Arikidis NS, Karahaliou A, Skiadopoulos S, Korfiatis P, Likaki E, Panayiotakis G, Costaridou L. Size-adapted microcalcification segmentation in mammography utilizing scale-space signatures. Computerized Medical Imaging and Graphics. 2010; 34(6):487-93. http://dx.doi.org/10.1016/j. compmedimag.2009.12.009

Azevedo MC. Manual de radiologia da mama. Rio de Janeiro: INCa, DuPont, Microservice; 1994.

Barlow WE. Performance of diagnostic mammography for women with signs or symptoms of breast cancer. Journal of the National Cancer Institute. 2002; 94(1):1151-9. http:// dx.doi.org/10.1093/jnci/94.15.1151

Calas MJG, Gutfilen B, Pereira WCA. CAD e mamografia: por que usar essa ferramenta? Radiologia Brasileira. 2012; 45(1):46-52. http://dx.doi.org/10.1590/ S0100-39842012000100011

Chen $\mathrm{CH}$, Lee GG. On digital mammogram segmentation and microcalcification detection using multiresolution wavelet analysis. Graphical Models and Image Processing. 1997; 59(5):349-64. http://dx.doi.org/10.1006/ gmip.1997.0443

Cheng HD, Cai X, Chen X, Hu L, Lou X. Computer-aided detection and classification of microcalcifications in mammograms: a survey. Pattern Recognition. 2003; 36(12):2967-91. http://dx.doi.org/10.1016/ S0031-3203(03)00192-4

De Santo M, Molinara M, Tortorella F, Vento M. Automated classification of clustered microcalcifications by a multiple expert system. Pattern Recognition. 2003; 36(1):1467-77. http://dx.doi.org/10.1016/S0031-3203(03)00004-9

Duarte, MA, Alvarenga, AV, Azevedo, CM, Infantosi, AFC, Pereira, WCA. Microcalcifications segmentation procedure based on morphological operators and histogram filtering. In: MEDICON2010: Proceedings of the 12nd Mediterranean Conference on Medical and Biological Engineering and Computing; 2010 May 27-30; Chalkidiki. Berlin: SpringerVerlag, 2010a. v. 29. p. 355-358.

Duarte MA, Alvarenga AV, Azevedo CM, Infantosi AFC, Pereira WCA. Segmenting microcalcifications by Tophat morphological operators and histogram filtering. In: CBEB 2010: Proceedings of the XXII Brazilian Congress on Biomedical Engineering; Nov 21-25 2010; Tiradentes. Rio de Janeiro: Soc. Bras. Eng. Biomédica; 2010b. p. 422-5.

Duarte MA, Alvarenga AV, Azevedo CM, Infantosi AFC, Pereira WCA. Automatic microcalcifications segmentation procedure based on Otsu's method and morphological filters. In: PAHCE 2011: Proceedings of Pan American Health Care Exchange; 2011 Mar 28 - Apr 1; Rio de Janeiro. Piscataway: IEEE; 2011. v. 1, p. 102-106.

Elter M, Horsch A. CADx of mammographic masses and clustered microcalcifications: A review. Medical Physics. 2009; 36(6):2052-68. http://dx.doi. org/10.1118/1.3121511

Fu JC, Lee SK, Wong STC, Yeh JY, Wang AH, Wu HK. Image segmentation feature selection and pattern classification for mammographic microcalcifications. Computerized Medical
Imaging and Graphics. 2005; 29(1):419-29. http://dx.doi. org/10.1016/j.compmedimag.2005.03.002

Gravielides MA, Lo JY, Floyd CEF Jr. Parameter optimization of a computer-aided diagnosis scheme for the segmentation of microcalcification clusters in mammograms. Medical Physics. 2002; 29(4):475-83. http:// dx.doi.org/10.1118/1.1460874

Halkiots S, Botsis T, Rangoussi M. Automatic detection of clustered microcalcifications in digital mammograms using mathematical morphology and neural networks. Signal Processing. 2007; 87(1):1559-68. http://dx.doi. org/10.1016/j.sigpro.2007.01.004

Instituto Nacional de Câncer - INCa. Tipos de Câncer, Mama [Internet]. Brasília: Ministério da Saúde; 2012a. [cited 2012 July 30]. Available from http://www2.inca. gov.br/wps/wcm/connect/tiposdecancer/site/home/mama.

Instituto Nacional de Câncer - INCa. Câncer de mama [Internet]. Brasília: Ministério da Saúde; 2012b. [cited 2012 July 30]. Available from http://www1.inca.gov.br/inca/ Arquivos/publicacoes/cancer_mama_2010.pdf.

Jalalian A, Mashoror SBT, Mahmudb HR, Saripan MIB, Ramli ARB, Karasfi B. Computer-aided detection/diagnosis of breast cancer in mammography and ultrasound: a review. Clinical Imaging. 2013; 37(1):420-6. http://dx.doi. org/10.1016/j.clinimag.2012.09.024

Mohanalin J, Kalra PK, Kumar N. Microcalcification segmentation using normalized Tsallis entropy: an automatic "q" calculation by exploiting type II fuzzy sets. IETE Journal of Research. 2009; 25(2):90-6. http://dx.doi. org/10.4103/0377-2063.53240

Moradmand H, Setayeshi S, Targhi HK. Comparing methods for segmentation of microcalcification clusters in digitized mammograms. IJCSI International Journal of Computer Science. $2011 ; 8(6): 104-8$.

Nishikawa RM. Current status and future directions of computer-aided diagnosis in mammography. Computerized Medical Imaging and Graphics. 2007; 31(1):224-35. http:// dx.doi.org/10.1016/j.compmedimag.2007.02.009

Otsu N. A threshold selection method from gray-level histograms. IEEE Transactions on Systems, Man, and Cybernetics. 1979; 9(1):62-66. http://dx.doi.org/10.1109/ TSMC.1979.4310076

Paquerault S, Yarusso LM, Papaioannou J, Jiang Y. Radial gradient-based segmentation of mammographic microcalcifications: observer evaluation and effect on CAD performance. Medical Physics. 2004; 31(9):2648-57. http:// dx.doi.org/10.1118/1.1767692

Stojic T, Reljin I, Reljin B. Local contrast enhancement in digital mammography by using mathematical morphology. In: ISSCS 2005: Proceedings of the International Symposium on Signals, Circuits and Systems; 2005; Iasi. Iasi; 2005. v. 2, n. 1, p. 609-12. http://dx.doi.org/10.1109/ISSCS.2005.1511314

Stojic T, Reljin I, Reljin B. Adaptation of multifractal analysis to segmentation of microcalcifications in digital mammograms. Physica A. 2006; 367(1):494-508. 
Timp S, Karssemeijer N. A new 2D segmentation method based on dynamic programming applied to computer aided detection in mammography. Medical Physics. 2004; 31(5):958-71. http://dx.doi.org/10.1118/1.1688039

University of South Florida. DOD Breast Cancer Research Program, US Army Research and Material Command, Digital Database for Screening Mammography - DDSM [Internet]. University of South Florida; 2012. [cited 2012 July 30]. Available from http://marathon.csee.usf.edu/ Mammography/Database.html.
Veldkamp WJ, Karssemeijer N, Otten JDM, Hendriks JHCL. Automated classification of clusters microcalcifications into malignant and benign types. Medical Physics. 2000; 27(11):2600-8. http://dx.doi. org/10.1118/1.1318221

Wei L, Yanga Y, Nishikawa RM. Microcalcification classification assisted by content-based image retrieval for breast cancer diagnosis. Pattern Recognit. 2009; 42(6):112632. http://dx.doi.org/10.1016/j.patcog.2008.08.028

\section{Authors \\ Marcelo de Almeida Duarte*, Antonio Fernando Catelli Infantosi, Wagner Coelho de Albuquerque Pereira Programa de Engenharia Biomédica, Instituto Alberto Luiz Coimbra - COPPE, Universidade Federal do Rio de Janeiro - UFRJ, CP 68510, CEP 21941-972, Rio de Janeiro, RJ, Brasil.}

\section{Marcelo de Almeida Duarte}

Electronic Engineering Department, Gama Filho University - UGF, Rio de Janeiro, RJ, Brazil.

\section{André Victor Alvarenga}

Laboratory of Ultrasound, National Institute of Metrology, Standardization and Industrial Quality - Inmetro, Rio de Janeiro, RJ, Brazil.

\section{Carolina Maria Azevedo}

Gaffrée \& Guinle University Hospital, University of Rio de Janeiro - UNI-RIO, Rio de Janeiro, RJ, Brazil.

\section{Maria Julia Gregório Calas}

Department of Radiology, School of Medicine, Federal University of Rio de Janeiro - UFRJ, Rio de Janeiro, RJ, Brazil. 